\title{
New exclusion limits on scalar and pseudoscalar axionlike particles from light shining through a wall
}

\author{
R. Ballou, ${ }^{1,2}$ G. Deferne, ${ }^{3}$ M. Finger, Jr., ${ }^{4}$ M. Finger, ${ }^{4}$ L. Flekova, ${ }^{5}$ J. Hosek, ${ }^{5}$ S. Kunc, ${ }^{6}$ K. Macuchova, ${ }^{5}$ K. A. Meissner, ${ }^{7}$ \\ P. Pugnat, ${ }^{8,2, *}$ M. Schott, ${ }^{9}$ A. Siemko, ${ }^{3}$ M. Slunecka, ${ }^{4}$ M. Sulc, ${ }^{6}$ C. Weinsheimer, ${ }^{9}$ and J. Zicha ${ }^{5}$ \\ (OSQAR Collaboration) \\ ${ }^{1}$ CNRS, Institut Néel, 38042 Grenoble, France \\ ${ }^{2}$ Université Grenoble Alpes, 38042 Grenoble, France \\ ${ }^{3}$ CERN, 1211 Geneva-23, Switzerland \\ ${ }^{4}$ Charles University, Faculty of Mathematics and Physics, 12116 Prague, Czech Republic \\ ${ }^{5}$ Czech Technical University, 16607 Prague, Czech Republic \\ ${ }^{6}$ Technical University of Liberec, 46117 Liberec, Czech Republic \\ ${ }^{7}$ University of Warsaw, Institute of Theoretical Physics, 00-681 Warsaw, Poland \\ ${ }^{8}$ CNRS, LNCMI, 38042 Grenoble, France \\ ${ }^{9}$ University of Mainz, Institute of Physics, 55128 Mainz, Germany
}

(Received 29 June 2015; published 2 November 2015)

\begin{abstract}
Physics beyond the Standard Model predicts the possible existence of new particles that can be searched at the low-energy frontier in the sub-eV range. The OSQAR photon regeneration experiment looks for "light shining through a wall" from the quantum oscillation of optical photons into "weakly interacting subeV particles," such as axion or axionlike particles (ALPs) in a $9 \mathrm{~T}$ transverse magnetic field over a length of $2 \times 14.3 \mathrm{~m}$. In 2014, this experiment was run with an outstanding sensitivity, using an $18.5 \mathrm{~W}$ continuous wave laser emitting in the green at the single wavelength of $532 \mathrm{~nm}$. No regenerated photons have been detected after the wall, pushing the limits for the existence of axions and ALPs down to an unprecedented level for such type of laboratory experiment. The diphoton couplings of possible pseudoscalar and scalar ALPs can be constrained in the nearly massless limit to be less than $3.5 \times$ $10^{-8} \mathrm{GeV}^{-1}$ and $3.2 \times 10^{-8} \mathrm{GeV}^{-1}$, respectively, at $95 \%$ confidence level.

\section{INTRODUCTION}

Possible extensions of the Standard Model (SM) of particle physics are not restricted to the high-energy frontier. There is also a growing interest for the search of weakly interacting sub-eV particles (WISPs) with much weaker interactions and masses below the eV. One emblematic example is the axion, a pseudoscalar boson arising from the spontaneous breaking of a global chiral symmetry $U(1)_{A}$ postulated to dynamically solve the strong $C P$ problem [1-3]. Axions and axionlike particles (ALPs) are predicted in supersymmetric theories [4], in string theory [5,6], and in the conformal Standard Model [7]. ALPs can be scalar as well as pseudoscalar and theorized to couple to the SM through a variety of mechanisms, giving rise, in particular, to a two-photon vertex. WISPs also include light bosons of gauge groups under which the SM particles are not charged (hidden sectors), which may

*pierre.pugnat@lncmi.cnrs.fr

Published by the American Physical Society under the terms of the Creative Commons Attribution 3.0 License. Further distribution of this work must maintain attribution to the author(s) and the published article's title, journal citation, and DOI. interact with the SM through gravity, kinetic mixing, or higher order quantum processes [8]. The interest aroused by WISPs goes beyond particle physics. As earlier hypothesized for the axion [9], they provide alternative candidates for dark matter [10-12]. Moreover, they might explain a number of astrophysical puzzles, such as the Universe transparency to very high-energy photons $(>100 \mathrm{GeV})$ [13], the anomalous white dwarf cooling [14], or the recently discovered gamma-ray excesses in galaxy clusters [15]. In contrast to weakly interacting massive particles, which can be searched for at $\mathrm{TeV}$ colliders such as the Large Hadron Collider (LHC) at CERN, the detection of WISPs requires to have recourse to dedicated low-energy experiments. Several methodologies exploiting the existence of a diphoton coupling have been proposed based on lasers, microwave cavities, strong electromagnetic fields, or torsion balances [16,17].

The OSQAR (Optical Search for QED Vacuum Birefringence, Axions, and Photon Regeneration) experiment at CERN is at the forefront of this low-energy frontier of particle/astroparticle physics. It combines the simultaneous use of high magnetic fields with laser beams in distinct experiments. One of its setups uses the "light shining through a wall" (LSW) method for the search of the 
ALPs [18]. A pioneering work in this line excluded ALPs with a diphoton coupling constant $g_{A \gamma \gamma}$ larger than $6.7 \times$ $10^{-7} \mathrm{GeV}^{-1}$ for masses below $10^{-3} \mathrm{eV}$ [19]. These exclusion limits were later extended by other LSW experiments to $g_{A \gamma \gamma}>6.5 \times 10^{-8} \mathrm{GeV}^{-1}$ for masses below $5 \times 10^{-4} \mathrm{eV}$ [20] and more recently tightened to $g_{A \gamma \gamma}>5.7 \times$ $10^{-8} \mathrm{GeV}^{-1}$ for masses below $2 \times 10^{-4} \mathrm{eV}$ [21]. New results are here reported, obtained from the 2014 data-taking campaign of the OSQAR LSW experiment. Compared to the previous experimental setups of OSQAR [21,22], a more powerful laser source and a detector with higher sensitivity have been used. In addition, an improved method of data analysis has been implemented.

\section{EXPERIMENTAL SETUP AND DATA TAKING}

LSW experiments are based on the combination of two factors. The first is the transparency of the WISPs to photons barriers, owing to the weakness of the interactions of the WISPs with the particles of the SM. The second is the photon-to-WISP and WISP-to-photon quantum oscillation, which would arise from the interactions of WISPs with photons $[18,23,24]$. In the case of the sub-eV ALPs, the method benefits from their two-photon vertex, inducing oscillations with optical photons in a transverse magnetic field. Such a field can be represented as a sea of virtual photons, whose interaction with real photons (respectively ALPs) can produce real ALPs (respectively photons). The mechanism is similar to the Primakov process of the production of neutral mesons by high-energy photons in a strong electric field [25]. The effective Lagrangian density of the interaction of an axion or a pseudoscalarALP (PS-ALP) field $\mathcal{A}$ with the electromagnetic field $F_{\mu \nu}$ is written generically in the form

$$
\mathcal{L}_{\text {int }}=-\frac{1}{4} g_{A \gamma \gamma} \mathcal{A} F_{\mu \nu} \tilde{F}^{\mu \nu}=g_{A \gamma \gamma} \mathcal{A} \vec{E} \cdot \vec{B}
$$

where $\tilde{F}^{\mu \nu}=\frac{1}{2} \epsilon^{\mu \nu \alpha \beta} F_{\alpha \beta}$ is the dual of $F_{\mu \nu}$, and the constant $g_{A \gamma \gamma}$ stands for the effective axion or ALP diphoton coupling. With a scalar-ALP (S-ALP) field $\mathcal{A}$, the interaction with the field $F_{\mu \nu}$ takes the generic form

$$
\mathcal{L}_{\text {int }}=-\frac{1}{4} g_{A \gamma \gamma} \mathcal{A} F_{\mu \nu} F^{\mu \nu}=g_{A \gamma \gamma} \mathcal{A} \frac{1}{2}\left(\vec{E}^{2}-\vec{B}^{2}\right) .
$$

Accordingly, either PS-ALPs or S-ALPs could potentially be created when a beam of linearly polarized photons propagates in a transverse magnetic field $\vec{B}$, depending on whether the polarization is parallel to the magnetic field $\left(\vec{E}_{\gamma} \| \vec{B}\right)$ or perpendicular $\left(\vec{E}_{\gamma} \perp \vec{B}\right)$. If an optical barrier is placed downstream to the beam, all unconverted photons will be absorbed while ALPs would traverse the optical barrier. By applying a second magnetic field in the regeneration domain beyond the wall, the inverse

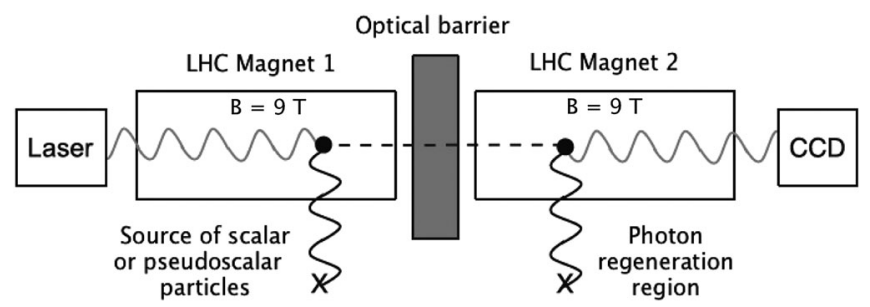

FIG. 1. Principle of the OSQAR LSW experiment.

Primakov process can convert the ALPs back into photons, which can be subsequently detected (Fig. 1). The probability of an ALP-to-photon $(A \rightarrow \gamma)$ or of a photon-to-ALP $(\gamma \rightarrow A)$ conversion is given by $[18,23,24]$

$$
P_{\gamma \leftrightarrow A}=\frac{1}{4}\left(g_{A \gamma \gamma} B L\right)^{2}\left(\frac{2}{q L} \sin \frac{q L}{2}\right)^{2}
$$

in units of heaviside-Lorentz system $(\hbar=c=1) . q=$ $\left|k_{\gamma}-k_{A}\right|$ stands for the momentum transfer, where $k_{\gamma}=\omega$ is the momentum of the photon of energy $\omega$ and $k_{A}=$ $\sqrt{\left(\omega^{2}-m_{A}^{2}\right)}$ the momentum of the ALP of mass $m_{A}$. The overall probability of the photon regeneration is $P_{\gamma \rightarrow A \rightarrow \gamma}=\left(P_{\gamma \leftrightarrow A}\right)^{2}$, as arising from the two consecutive conversions: $\gamma \rightarrow A$ followed by $A \rightarrow \gamma$. The flux of the regenerated photons to detect is then given by

$$
\frac{d N}{d t}=\frac{P}{\omega} \eta\left(P_{\gamma \leftrightarrow A}\right)^{2}
$$

where $P$ represents the power of the incoming photon beam, and $\eta$ stands for the photon detection efficiency. $d N / d t$ is proportional to the fourth power of the field integral $B L$, whence the need for the strongest magnetic field $B$ over longest optical path $L$ to achieve highest sensitivity.

The OSQAR LSW experiment uses two spare LHC dipole magnets - the first for the ALPs production and the second for the photon regeneration. Each magnet is cooled down to $1.9 \mathrm{~K}$ with superfluid $\mathrm{He}$ and provides a uniform transverse magnetic field with a strength of $9 \mathrm{~T}$ over a magnetic length of $14.3 \mathrm{~m}$, thus, giving rise to a magnetic field integral of $B L=128.7 \mathrm{Tm}$. The aperture of both magnets have been pumped, using turbo-molecular pumping groups, down to $10^{-5}-10^{-7}$ mbar.

A continuous wave optical power of $18.5 \mathrm{~W}$ at a single wavelength of $532 \mathrm{~nm}(2.33 \mathrm{eV})$ was delivered by a diodepumped solid-state laser (Verdi V18 from Coherent, Inc.). A beam expander telescope was used to reduce the laser beam divergence. The photon beam is linearly polarized with a vertical orientation parallel to the magnetic field for the search of PS-ALPs. A $\lambda / 2$ wave plate with antireflective (AR) coating layers was inserted at the laser exit in order to align the photon polarization in the perpendicular direction for the search of S-ALPs. 
The laser beam at the exit of the second magnet aperture was focused by an optical lens on a thermoelectric cooled charge-coupled device (CCD) with an AR-coated window (DU934P-BEX2-DD from Andor Technology Ltd.). The CCD chip has an active area of $13.3 \times 13.3 \mathrm{~mm}^{2}$ and is composed by a 2D array of $1024 \times 1024$ square pixels of size $13 \times 13 \mu \mathrm{m}^{2}$. The laser beam was focused at $95 \%$ on an area covering not more than four pixels. The CCD was cooled down to the temperature range $\left[-95^{\circ} \mathrm{C},-92^{\circ} \mathrm{C}\right]$. The dark current is $0.0012 e^{-} / \mathrm{pixel} / \mathrm{s}$ at $-95^{\circ} \mathrm{C}$ and $0.0020 e^{-} / \mathrm{pixel} / \mathrm{s}$ at $-92^{\circ} \mathrm{C}$. The read-out noise was determined at $2.5 e^{-} \mathrm{rms} /$ pixel from frames recorded in minimal time with closed shutters. These measured CCD parameters are in agreement with those provided by the manufacturer. The overall photon detection efficiency was explicitly measured to $\eta=0.56 \pm 0.02$ analog-to-digital unit (ADU)/photon, taking into account the losses coming from optical elements including windows, plates, lens, CCD quantum efficiency of 0.88 at $532 \mathrm{~nm}$, and sensitivity of $1.3 e^{-} /$ADU. The total optical power loss of the whole optical setup is, thus, approximately $17 \%$, in good agreement with the characteristics of the various optical elements in use.

The data taking for the ALPs search reported in this paper was performed in August 2014 and corresponds to a total of 119 experimental runs, as detailed in Table I.

Each experimental run was composed of two frames of $5400 \mathrm{~s}$ exposure time separated by a $100 \mathrm{~s}$ pause. The recording time for a single frame was defined to optimize the signal-to-noise ratio, taking into account the filtering and removal procedure of cosmic-ray signatures. Before and after each run, the position of the laser beam was precisely measured on the CCD with the optical power strongly reduced. Starting with a laser beam power settled to $3 \mathrm{~W}$, a variable beam splitter was introduced to attenuate the beam typically by a factor of $1 / 500$. To carefully check the stability of the laser beam spot, three frames were recorded each time with $0.01 \mathrm{~s}$ exposure separated by a 120 s pause. Subsequently, the optical barrier was introduced, and the attenuator was settled to its minimum before ramping up the laser power to $18.5 \mathrm{~W}$. If the experimental conditions were not optimal or if cosmic rays impacted the signal region, then the experimental run was rejected (Table I). The signal region is defined as the pixels of the CCD where reconverted photons are expected to be

TABLE I. Summary of the experimental runs. $\vec{E}_{\gamma}$ stands for the electric component of the linearly polarized photon field, $\vec{B}$ the static magnetic field, $P$ the laser power, $N_{T}$ the number of recorded runs, and $N_{R}$ the number of rejected runs. W (95\%) is the Bayesian threshold of nondetection at $95 \%$.

\begin{tabular}{lcccccc}
\hline \hline Search for & $\vec{E}_{\gamma}$ & $\vec{B}$ & $P$ & $N_{T}$ & $N_{R}$ & W (95\%) \\
\hline PS-ALPs & $\| \vec{B}$ & $9 \mathrm{~T}$ & $18.5 \mathrm{~W}$ & 59 & 18 & $0.64 \mathrm{mHz}$ \\
S-ALPs & $\perp \vec{B}$ & $9 \mathrm{~T}$ & $18.5 \mathrm{~W}$ & 60 & 12 & $0.45 \mathrm{mHz}$ \\
\hline \hline
\end{tabular}

detected, taking into account slight displacements of the laser-beam spot.

\section{DATA REDUCTION}

The detailed analysis of all beam positions during the full data-taking period clearly confirmed that environmental temperature variations induce only minor low-frequency displacement/deformation of the CCD support, and, therefore, only a small relative shift of the beam spot on the detector, typically less or equal to one pixel per hour. The beam spot displacement is unidirectional, with no oscillation observed after the exposure time of a full run, i.e., after $2 \times 5400 \mathrm{~s}+100 \mathrm{~s}=10900 \mathrm{~s}$. The beam spot sizes before and after each experimental run are obtained from least-squares fits with a two-dimensional Gaussian distribution. To define conservatively the signal region, twice the maximum value of the Gaussian widths computed from the intitial $(i)$ and final $(f)$ reference frames are retained, i.e., $2 \sigma_{\text {run }}=2 \times \max \left[\sigma_{i}, \sigma_{f}\right]$. If the laser positions before and after a run do not coincide, all pixels within a $2 \sigma_{\text {run }}$ orthogonal distance along a straight line between the final and initial positions are added to the signal region. An illustrative example is provided in Fig. 2. It follows that the number $S$ of pixels that defines the signal region can vary from run to run (Table II). A cumulated count $N_{i}$ can be associated to each run- $i$, by adding the counts collected during the exposure time of $2 \times 5400 \mathrm{~s}$ for ALPs detection on all the pixels of the signal region, once this one is carefully determined. $N_{i}$ is the relevant quantity that would reveal the regenerated photons if it is proven in excess over background noises.

One of the advantages of a small and well-defined signal region is the possibility to use the remaining pixels, which are not exposed to possible ALP signals, to fully characterize the background without repeating dedicated background acquisition runs [21,22]. These remaining pixels are clustered in size and shape that correspond to the signal region. Cumulated counts $n_{i j}$ are obtained for each cluster $j$ in the background region of each run- $i$, by adding the counts collected on all the pixels of the chosen cluster, similar to the cumulated count $N_{i}$ of the signal region. The

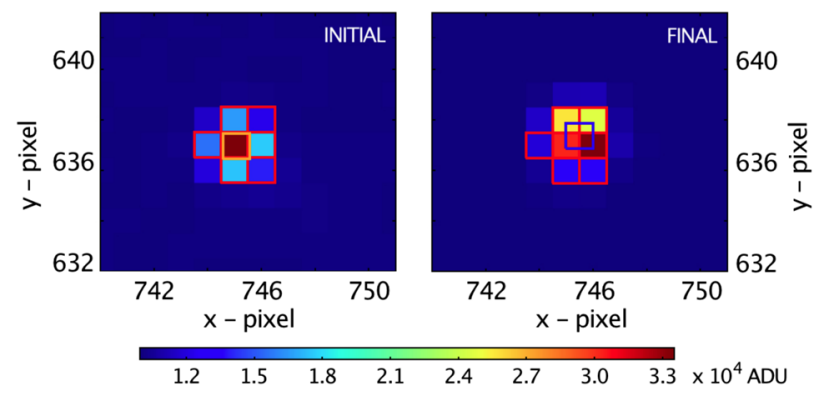

FIG. 2 (color online). Initial and final positions of the attenuated laser beam on the CCD for run-90 devoted to the search of S-ALPs $\left(\vec{E}_{\gamma} \perp \vec{B}\right)$. The signal region covers seven pixels. 
TABLE II. Distribution of sizes $S$ in units of pixels of the signal regions. $N_{\mathrm{PS}}$ (respectively $N_{S}$ ) counts the number of runs devoted to the search of PS-ALPs (respectively S-ALPs) for which the same given size $S$ was experimentally deduced for the signal region.

\begin{tabular}{lccccccccccccccc}
$S$ & 6 & 7 & 8 & 9 & 10 & 11 & 12 & 13 & 14 & 15 & 16 & 17 & 18 & 19 & 20 \\
\hline$N_{\text {PS }}$ & 0 & 2 & 6 & 4 & 3 & 4 & 7 & 4 & 3 & 2 & 0 & 2 & 3 & 0 & 1 \\
$N_{S}$ & 6 & 5 & 6 & 11 & 3 & 4 & 5 & 3 & 2 & 1 & 1 & 0 & 0 & 0 & 1
\end{tabular}

\begin{tabular}{llllllllllllllll}
$N_{S}$ & 6 & 5 & 6 & 11 & 3 & 4 & 5 & 3 & 2 & 1 & 1 & 0 & 0 & 0 & 1 \\
\hline
\end{tabular}

distribution of the $n_{i j}$ for a given run- $i$ then provides an estimate of the expected background contribution to $N_{i}$. As it is well known for CCDs, the width of this distribution arises mainly from two independent sources, namely, dark counts that increase with exposure times and read-out noise that arises from the analog-digital conversion process of the read-out system.

Before analyzing the cumulative counts, three corrections have been applied to each recorded frame pair of $2 \times 5400 \mathrm{~s}$. The first one aims to correct long wavelength distortions of the spatial distribution of the background arising from the minor heterogenity in the cooling of the CCD chip. Corrections of these background drifts are achieved by median filtering. The objective of the second correction is to reduce the so-called fixed pattern noise evolving from possibly unequal pixel biases. As this noise manifests only during read-out and is independent of the exposure time, it has been estimated by capturing dark frames (i.e., shutter closed) with the minimal exposure time $\left(10^{-5} \mathrm{~s}\right.$ in our case). Ten frames have been recorded under these conditions and have been averaged pixelwise to define a bias frame, which is subtracted from every frame

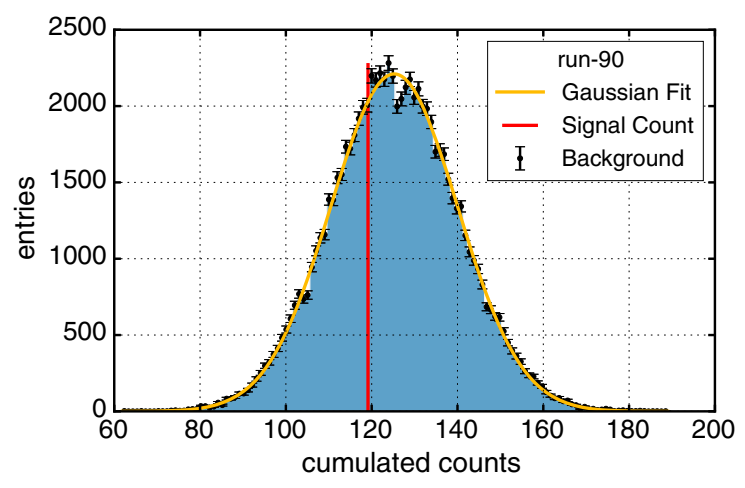

FIG. 3 (color online). Distribution of the cumulated counts $n_{90 j}$ of background clusters $j$ of size and shape identical to the signal region shown Fig. 2 for run-90, after applying all background corrections (see text). Gaussian fit gives mean $\mu_{90}^{\text {bkg }}=125.24 \pm$ 0.05 and standard deviation $\sigma_{90}^{\mathrm{bkg}}=14.77 \pm 0.04$ with reduced chi-squared $\chi^{2} /$ n.d.f. $=1.04$. The red line represents the cumulated count $N_{90}=119$ of the signal region. It crosses the Gaussian distribution at about 2000, which corresponds to the number of background clusters with the same cumulated count as the signal region. used in the data analyses. This correction also subtracts the general constant offset of about 3300 counts on each pixel imprinted by the chip in each frame of $5400 \mathrm{~s}$. The last correction step removes those pixels, which were hit by cosmic rays, using a short wavelength filtering process. Whenever a pixel count exceeds the significant threshold of the distribution of pixel counts in the background region, this pixel is rejected together with its nearest neighbors. In case the signal region is hit by a cosmic ray, the overall frame is rejected.

Once all correction steps have been applied to a given run- $i$, the cumulated counts $N_{i}$ in the signal region and $n_{i j}$ of the clusters $j$ in the background region are evaluated. As illustrated in Fig. 3 for the run-90, the distribution of the cumulated counts $n_{i j}$ for every run- $i$ is a Gaussian with mean $\mu_{i}^{\mathrm{bkg}}$ and standard deviation $\sigma_{i}^{\mathrm{bkg}}$ in good agreement with the total noise of the CCD expected from dark counts and read-out noise contributions.

\section{BAYESIAN ANALYSIS}

A detection limit on the rate of reconverted photons $d N / d t$ can be extracted from the Gaussian width of cumulated background cluster counts over the pixelwise sum of all the frames for a given photon polarization. This requires choosing a signal region of the same size for all the runs, which necessarily must be the largest one that is $S=20$ pixels according to Table II. A slightly better detection limit can be obtained by rejecting the few runs with a too-large size of signal region but at the statistical cost of the counts inherent to the corresponding decrease of the total exposure time. This strategy used in previously published analyses of similar experiments, e.g., Ref. [22], is not optimal. A detection limit at 2 times the standard deviation of the background fluctuations in the order of $2 \mathrm{mHz}\left(2 \times 10^{-3} \mathrm{ADU} \mathrm{s}^{-1}\right)$ can be obtained with this approach. This does not constitute a real improvement with respect to a single run with a smaller size of signal region such as run-90 for which $S=7$ pixels, because the increase in cluster size leads to larger background fluctuations for such a run.

In order to optimize the detection limit by considering real signal regions of different sizes from run to run (Table II), a Bayesian approach has been implemented (cf. Ref. [26], pages 472-487). The following likelihood model has been considered

$$
\mathcal{L} \propto \prod_{i} \mathcal{N}\left(N_{i} \mid \mathcal{P}\left(\frac{d N}{d t} t_{i}^{\mathrm{exp}}\right)+\mu_{i}^{\mathrm{bkg}}, \sigma_{i}^{\mathrm{bkg}}\right)
$$

with the rate of reconverted photons $d N / d t$ as the signal parameter. The index $i$ iterates over the integer number of runs corresponding to a certain polarization state, and $\mathcal{N}$ represents the Gaussian background parametrization of the $i$ th frame pair including an additional Poissonian signal 
NEW EXCLUSION LIMITS ON SCALAR AND ...
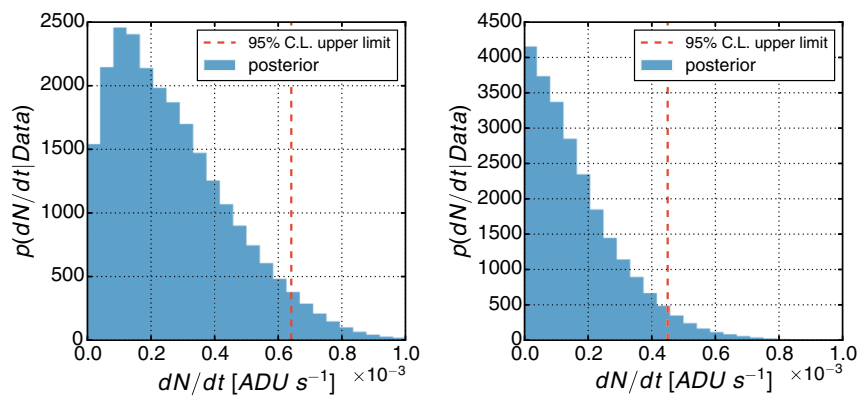

FIG. 4 (color online). Posterior probability distribution function $p(d N / d t \mid$ data $)$ of reconverted photon rate $d N / d t$ for incoming photon polarization parallel $\left(\vec{E}_{\gamma} \| \vec{B}\right)$ (left) and perpendicular $\left(\vec{E}_{\gamma} \perp \vec{B}\right)$ (right) to the magnetic field $\vec{B}$.

contribution $\mathcal{P}\left(\frac{d N}{d t} t_{i}^{\exp }\right)$ of expectation value $d N / d t$, multiplied by the frame pair exposure time $t_{i}^{\exp }=2 \times 5400 \mathrm{~s}$. Equation (5) allows for run-to-run variations in cluster size characterized by mean background counts $\mu_{i}^{\mathrm{bkg}}$ and associated standard deviations $\sigma_{i}^{\mathrm{bkg}}$ as in the typical Gaussian shown in Fig. 3. The numerical integration was performed via Markov chain Monte Carlos using $20 \times 10^{6}$ samples for each photon polarization state, leading to a negligible uncertainty due to the integration process.

The posterior probability distribution function $p(d N / d t \mid$ data $)$ of reconverted photon rate $d N / d t$ deduced
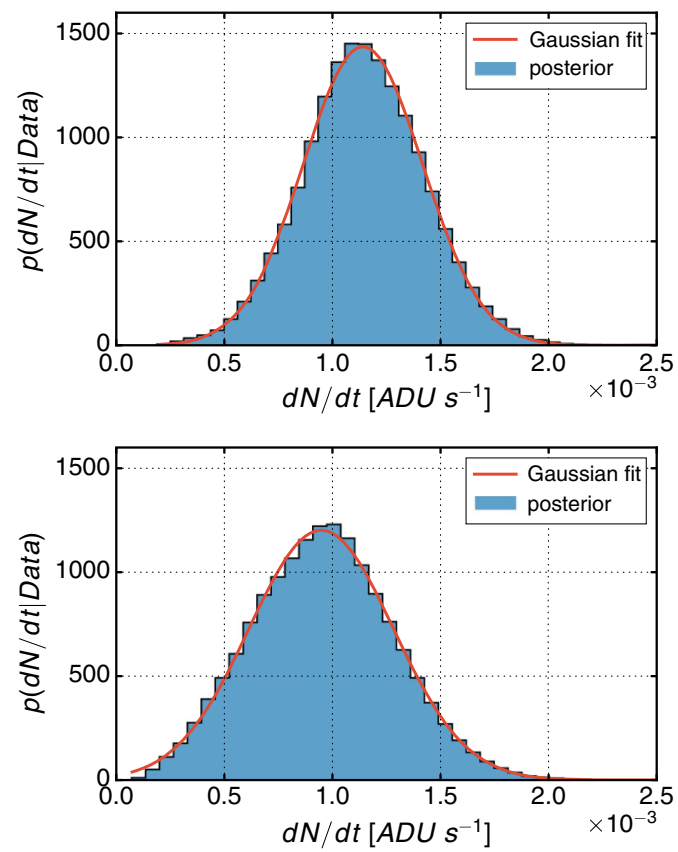

FIG. 5 (color online). Posterior probability distribution function $p(d N / d t \mid$ data $)$ of reconverted photon rate $d N / d t$ for incoming photon polarization parallel $\left(\vec{E}_{\gamma} \| \vec{B}\right)$ (top) and perpendicular $\left(\vec{E}_{\gamma} \perp \vec{B}\right)$ (bottom) to the magnetic field $\vec{B}$, with an artificially imposed fake signal of $1 \times 10^{-3} \mathrm{ADU} \mathrm{s}^{-1}$.
PHYSICAL REVIEW D 92, 092002 (2015)

from the considered likelihood model assuming a flat prior distribution for the signal parameter $d N / d t$ is displayed in Fig. 4 for the two linear polarizations of the incoming photon beam.

The consistency of the overall data analysis including filtering and statistical methods was checked by imposing into all selected raw data frames fake signals with a rate of $1 \mathrm{mHz}$ corresponding to a hypothetical ALP with a diphoton coupling constant $g_{A \gamma \gamma}=3.8 \times 10^{-8} \mathrm{GeV}^{-1}$ in the nearly massless limit $\left(m_{A} \rightarrow 0\right)$. A flux of reconverted photons $d N / d t=1.14 \pm 0.28 \mathrm{mHz}$ is then inferred from the posterior probability distribution function $p(d N / d t \mid$ data $)$ for incoming photons linearly polarized parallel to the magnetic field and $d N / d t=0.95 \pm$ $0.33 \mathrm{mHz}$ for incoming photons linearly polarized perpendicular to the magnetic field (Fig. 5).

\section{EXCLUSION LIMITS}

As a conclusion, no significant excess over the background expectation has been observed in the signal regions for the parallel or perpendicular polarization. Exclusion limits on the diphoton coupling strength $g_{A \gamma \gamma}$ and the ALPs mass $m_{A}$ have then been derived from Eqs. (3) and (4).

The 95\% confidence limit (C.L.) on the reconverted photon flux is derived via the posterior distribution of the signal parameter $d N / d t$ (Fig. 4). The results for the PS-ALPs and S-ALPs searches are summarized in Fig. 6, which also shows the exclusion limits reported by the ALPS Collaboration [20]. A limit for the diphoton couplings of $g_{A \gamma \gamma}<3.5 \times 10^{-8} \mathrm{GeV}^{-1}$ and $g_{A \gamma \gamma}<3.2 \times$ $10^{-8} \mathrm{GeV}^{-1}$ is obtained for the pseudoscalar and scalar searches for $m_{A}<2 \times 10^{-4} \mathrm{eV}$, respectively. These results establish the most stringent constraints on ALP searches in the nearly massless limit obtained so far by LSW experiments.

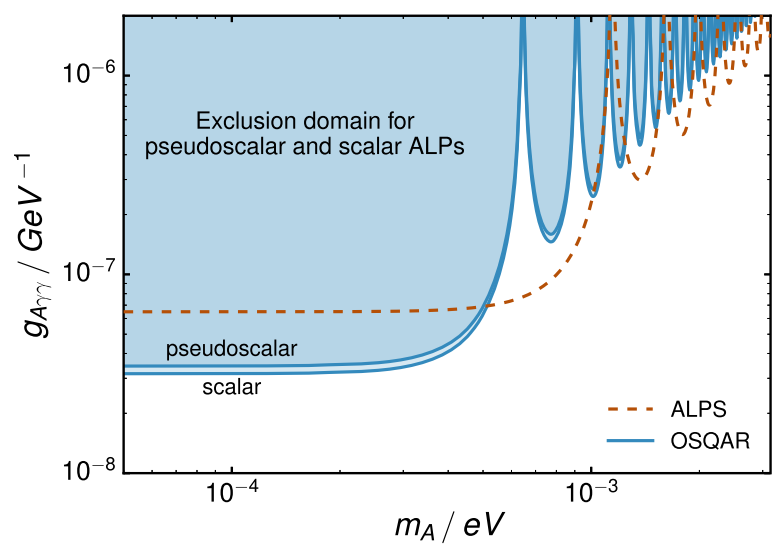

FIG. 6 (color online). Exclusion limits for the searches of PSALPs and S-ALPs at 95\% C.L. obtained in vacuum by the present OSQAR LSW experiment. Also reported for comparison is the latest result of ALPS LSW experiment in vacuum. 


\section{ACKNOWLEDGMENTS}

This work was partly supported by the Czech Science Foundation, Grant No. 203/11/1546, by the Students Grant
No. 201521053 of the Technical University of Liberec, and by the Cluster of Excellence PRISMA at the University of Mainz.
[1] R. D. Peccei and Helen R. Quinn, Phys. Rev. Lett. 38, 1440 (1977).

[2] S. Weinberg, Phys. Rev. Lett. 40, 223 (1978).

[3] F. Wilczek, Phys. Rev. Lett. 40, 279 (1978).

[4] L. Covi, J. E. Kim, and L. Roszkowski, Phys. Rev. Lett. 82, 4180 (1999).

[5] P. Svrcek and E. Witten, J. High Energy Phys. 06 (2006) 051.

[6] M. Cicoli, M. Goodsell, and A. Ringwald, J. High Energy Phys. 12 (2012) 146.

[7] K. A. Meissner and H. Nicolai, Phys. Lett. B 660, 260 (2008).

[8] B. Holdom, Phys. Lett. 166B, 196 (1986).

[9] L. F. Abbott and P. Sikivie, Phys. Lett. 120B, 133 (1983).

[10] R. Bradlay, J. Clarke, D. Kinion, L. J. Rosenberg, K. van Bibber, S. Matsuki, M. Mück, and P. Sikivie, Rev. Mod. Phys. 75, 777 (2003).

[11] P. Arias, D. Cadamuro, M. Goodsell, J. Jaeckel, J. Redondo, and A. Ringwald, J. Cosmol. Astropart. Phys. 06 (2012) 013.

[12] A. Ringwald, Phys. Dark Univ. 1, 116 (2012).

[13] M. Meyer, D. Horns, and M. Raue, Phys. Rev. D 87, 035027 (2013).
[14] M. M. M. Bertolami, B. E. Melendez, L. G. Althaus, and J. Isern, J. Cosmol. Astropart. Phys. 10 (2014) 069.

[15] M. Cicoli, J. P. Conlon, M. C. David Marsh, and M. Rummel, Phys. Rev. D 90, 023540 (2014).

[16] J. Jaeckel and A. Ringwald, Annu. Rev. Nucl. Part. Sci. 60, 405 (2010).

[17] R. Essig et al., arXiv:1311.0029.

[18] K. Van Bibber, N. R. Dagdeviren, S. E. Koonin, A. K. Kerman, and H. N. Nelson, Phys. Rev. Lett. 59, 759 (1987).

[19] R. Cameron et al., Phys. Rev. D 47, 3707 (1993).

[20] K. Ehret et al. (ALPS Collaboration), Phys. Lett. B 689, 149 (2010).

[21] R. Ballou et al. (OSQAR Collaboration), arXiv: $1410.2566 \mathrm{v} 1$.

[22] P. Pugnat et al. (OSQAR Collaboration), Eur. Phys. J. B 74, 3027 (2014).

[23] P. Sikivie, Phys. Rev. Lett. 51, 1415 (1983).

[24] P. Arias, J. Jaeckel, J. Redondo, and A. Ringwald, Phys. Rev. D 82, 115018 (2010).

[25] H. Primakov, Phys. Rev. 81, 899 (1951).

[26] K. A. Olive et al. (Particle Data Group), Chin. Phys. C 38, 090001 (2014). 